\title{
Telomere protein Rap1 is a charge resistant scaffolding protein in chromosomal bouquet formation
}

\author{
Hanna Amelina, Shaan Subramaniam, Vera Moiseeva, Christine Anne Armstrong, Siân Rosanna Pearson \\ and Kazunori Tomita ${ }^{*}$ (i)
}

\begin{abstract}
Background: Chromosomes reorganize in early meiotic prophase to form the so-called telomere bouquet. In fission yeast, telomeres localize to the nuclear periphery via interaction of the telomeric protein Rap1 with the membrane protein Bqt4. During meiotic prophase, the meiotic proteins Bqt1-2 bind Rap1 and tether to the spindle pole body to form the bouquet. Although it is known that this polarized chromosomal arrangement plays a crucial role in meiotic progression, the molecular mechanisms of telomere bouquet regulation are poorly understood.

Results: Here, we detected high levels of Rap1 phospho-modification throughout meiotic prophase, and identified a maximum of 35 phosphorylation sites. Concomitant phosphomimetic mutation of the modification sites suggests that Rap1 hyper-phosphorylation does not directly regulate telomere bouquet formation or dissociation. Despite the negative charge conferred by its highly phosphorylated state, Rap1 maintains interactions with its binding partners. Interestingly, mutations that change the charge of negatively charged residues within the Bqt1-2 binding site of Rap1 abolished the affinity to the Bqt1-2 complex, suggesting that the intrinsic negative charge of Rap1 is crucial for telomere bouquet formation.

Conclusions: Whereas Rap1 hyper-phosphorylation observed in meiotic prophase does not have an apparent role in bouquet formation, the intrinsic negative charge of Rap1 is important for forming interactions with its binding partners. Thus, Rap1 is able to retain bouquet formation under heavily phosphorylated status.
\end{abstract}

Keywords: Telomere, Shelterin complex, Schizosaccharomyces pombe, Phosphorylation, Meiosis

\section{Background}

Telomeres are specialized nucleoprotein structures that form the natural ends of linear chromosomes. While telomeres are mostly known for their essential function in chromosome maintenance, they also play an important role in meiotic progression [1]. During meiotic prophase, the position of chromosomes within the nucleus is dramatically reorganized and telomeres cluster within a limited area of the nuclear envelope to form the so-called telomere bouquet $[2,3]$. This conserved reorganization of chromosomes during meiotic prophase has been observed in most eukaryotic organisms and is shown to promote homolog pairing and meiotic recombination [4]. Recent

\footnotetext{
* Correspondence: k.tomita@ucl.ac.uk

Chromosome Maintenance Group, UCL Cancer Institute, University College London, London WC1E 6DD, UK
}

C Biomed Central

(c) 2015 Amelina et al. This is an Open Access article distributed under the terms of the Creative Commons Attribution License (http://creativecommons.org/licenses/by/4.0), which permits unrestricted use, distribution, and reproduction in any medium, provided the original work is properly credited. The Creative Commons Public Domain Dedication waiver (http:// creativecommons.org/publicdomain/zero/1.0/) applies to the data made available in this article, unless otherwise stated. in meiotic spindle formation [5].

In fission yeast Schizosaccharomyces pombe, the telomere bouquet is observed throughout meiotic prophase. This period is also known as the 'horsetail nucleus' stage, during which the nucleus elongates and oscillates back and forth between the cell poles, following the spindle pole body or SPB (the yeast equivalent of the centrosome) driven by cytoplasmic microtubules [6]. Bouquet formation is achieved by expression of a pair of meiosisspecific proteins, Bqt1 and Bqt2, which bridge the telomeric proteins Taz1 and Rap1 to the SPB component Sad1 [7]. To ensure telomere attachment to the SPB, telomeres must be tethered to the nuclear envelope via the interaction between Rap1 and the inner nuclear membrane complex Bqt3 and Bqt4 [8]. Disruption of any of 
these components leads to failure of telomere clustering and defective spore formation in meiosis [7-11]. Sporulation defects in the bouquet mutants occur mainly due to impaired spindle formation and partly due to compromised meiotic centromere assembly, followed by chromosome segregation defects $[5,12]$. Recent studies suggest that recruitment of a single telomere tract or centromere to the SPB is sufficient to confer functional spindles $[13,14]$. Hence, the telomere bouquet does not only function in alignment of chromosomes but is also crucial for the recruitment of a chromosome to the SPB to create a functional meiotic spindle.

Although the bouquet composition and its function are becoming better understood, the molecular mechanisms that govern dissociation of telomeres from the SPB remain elusive. Interestingly, in fission yeast, polarized bouquet configuration is maintained until the end of meiotic prophase, and upon entry into the first meiotic division, telomeres dissociate from the SPB in a concerted manner, dubbed 'telomere fireworks' [5]. Another interesting observation is that Bqt1 and Bqt2 do not localize to telomeres at the onset of the first meiotic division [7]. Moreover, Rap1 is highly phosphorylated in mitotic cells [15]. We therefore hypothesized that disruption of the interaction between Rap1 and the Bqt1-2 complex, potentially by means of post-translational modifications of one or both interacting partners, may be responsible for telomere dissociation from the SPB. In this report, we investigated whether Rap1 is involved in the termination of the telomere bouquet. Comprehensive phosphoproteomic analysis of the meiotic Rap1 protein revealed that it is progressively phosphorylated throughout meiotic prophase and the number of phosphosites peaks after completion of the bouquet stage. Surprisingly, this massive phosphorylation of Rap1 is dispensable for telomere bouquet dissociation, as indicated by our live microscopy analysis and protein interaction studies of the phospho-mutants. Our study illuminates that Rap1 is a protein resistant to negative charge and functions as a 'scaffolding' protein in the telomere bouquet.

\section{Results}

\section{Rap1 is hyper-phosphorylated in meiosis}

To investigate the stability of the Rap1 protein throughout meiosis, a homozygous diploid $\left(h^{-} / h^{-}\right)$temperaturesensitive pat1-114 strain carrying a mat-Pc cassette was utilized to synchronize meiosis [16, 17]. Meiosis was induced after nitrogen starvation, followed by a temperature shift from permissive $\left(26^{\circ} \mathrm{C}\right)$ to restrictive conditions $\left(34^{\circ} \mathrm{C}\right)$ (Fig. 1a). Progression of meiosis was monitored by assessing the number of nuclei and DNA content per cell from fractions collected at 30-minute or 1-hour intervals during the synchronization procedure (Fig. 1b). In order to assess Rap1 protein stability during meiosis, Rap1 was endogenously tagged with PK (V5) epitope peptide and detected by anti-V5 antibodies. Western blotting analysis of synchronous culture extracts showed that Rap1 protein is rather stably expressed during meiosis, although lower molecular weight, potentially truncated forms of Rap1, were observed at the end of meiosis (Fig. 1c, top panel).

Interestingly, a number of distinctly shifted bands of Rap1 were detected during meiotic prophase. Similar shifted bands of Rap1 have also been recently reported [18]. To determine if Rap1 is phosphorylated during meiosis, cell extracts were further analyzed using Phos$\operatorname{tag}^{\text {mix }}$ SDS-PAGE [19]. Phos-tag ${ }^{\text {Tw }}$ gel analysis revealed that the Rap1 protein is highly phosphorylated during meiosis. Strikingly, the maximum level of phosphorylation was observed at $4.5-5 \mathrm{hr}$, when almost none of the fastmigrating forms of Rap1 were detected (Fig. 1d). Phosphatase treatment confirmed that the shifted bands observed at 4.5-5 hr represented phosphorylated forms of Rap1 (Fig. 1e). Thus, our data indicates that Rap1 phosphorylation accumulates as meiotic prophase progresses, and Rap1 becomes hyper-phosphorylated at the onset of meiosis I, when the bouquet stage ends [5].

\section{Mass spectrometry analysis of Rap1 reveals an increasing number of phosphosites detected upon completion of the bouquet stage}

To determine the location of phosphorylation sites in Rap1, meiotic Rap1 was purified from fractions of the synchronized culture at $3.5 \mathrm{hr}$ and $4.5 \mathrm{hr}$, and was subjected to mass spectrometry analysis. Using trypsin digestion, we covered $70-75 \%$ of the Rap1 protein sequence at $95 \%$ peptide threshold, and identified 19 and 35 phosphorylation sites from $3.5 \mathrm{hr}$ and $4.5 \mathrm{hr}$, respectively (Additional file 1). Notably, all phosphorylated sites identified at $3.5 \mathrm{hr}$ were also detected at $4.5 \mathrm{hr}$, suggesting that Rap1 phosphorylation accumulates with progression of meiotic prophase. Our analysis revealed several meiosis-specific phosphorylation sites in addition to those detected in mitosis-arrested cells [15]. With respect to known protein binding domains of Rap1 $[15,20]$, the phosphorylated sites at $3.5 \mathrm{hr}$ (early prophase) fell into three clusters, whereas at $4.5 \mathrm{hr}$ phosphosites were fairly evenly distributed across Rap1 (Fig. 2). Interestingly, two and six phosphosites were identified in the Bqt1-2 binding area (311-370 amino acids) at $3.5 \mathrm{hr}$ and $4.5 \mathrm{hr}$, respectively. Notably, although phosphorylations were detected within the Bqt4 and Pozl binding regions, we did not detect any phosphorylation within known structural domains of Rap1. Altogether, our mass spectrometry data suggest that the number of phosphorylated residues of Rap1 increases with the progression of meiosis, which is in agreement with our Phos-tag gel analysis (Fig. 1d). 


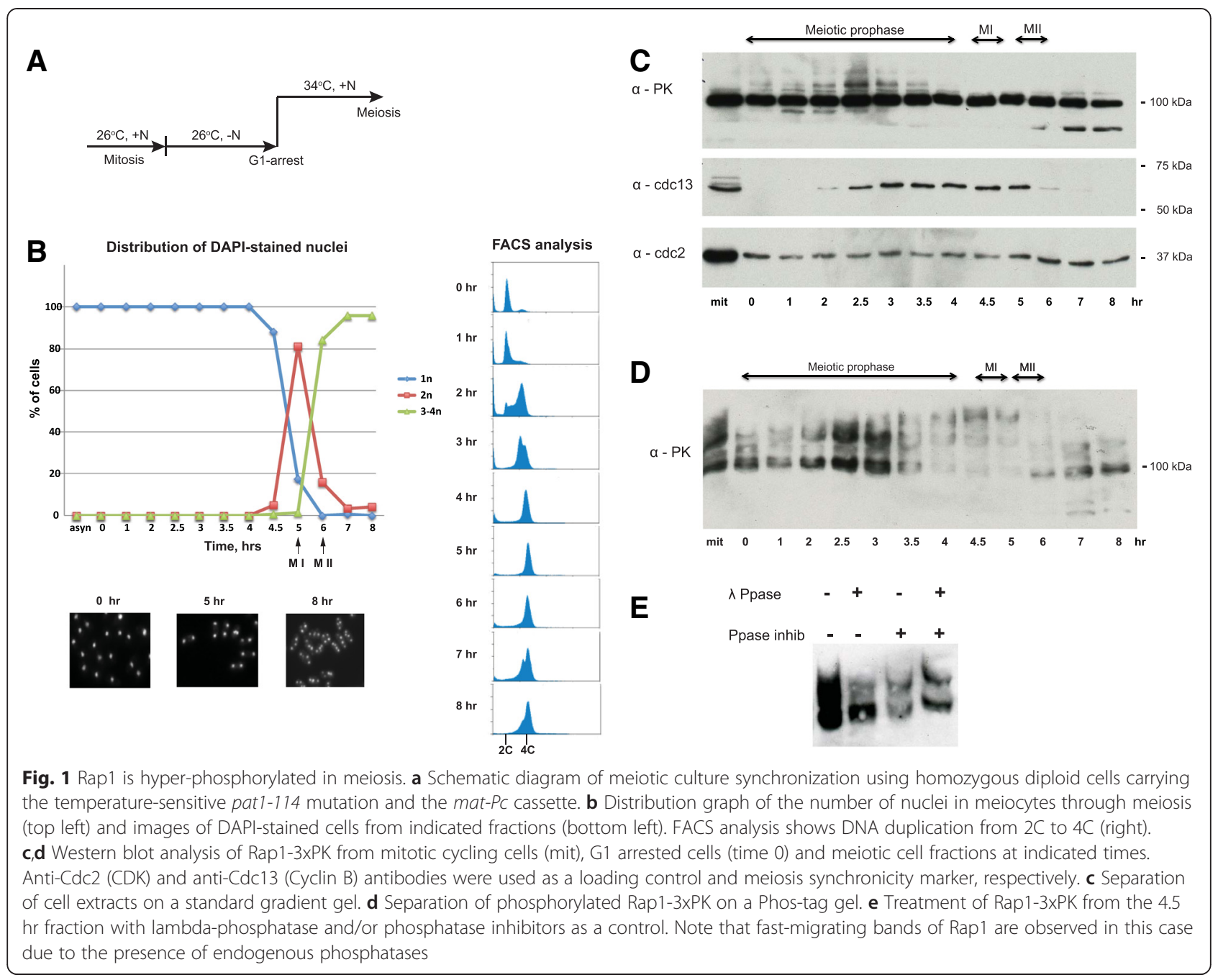

Hyper-phosphorylation of Rap1 in meiosis is dispensable for telomere bouquet formation and dissociation

Since we observed that Rap1 phosphorylation peaks at meiosis I, we speculated that the resulting highly negative charge of Rap1 is responsible for the change in its affinity to the Bqt1-2 complex. In order to mimic hyperphosphorylated Rap1, all validated phosphorylation sites from S-212 to S-562 were substituted with negatively charged glutamate residues (rap1-32E) (Fig. 3a). To monitor telomeres and the SPB through meiosis, endogenous Taz1 and Sid4 were tagged with YFP and mCherry, respectively. To our surprise, the phosphomimetic rap1-32E mutants did not exhibit any detectable meiotic defects and their telomeres clustered and dissociated from the SPB in a timely manner very similar to that of the wild-type (Fig. 3b). Accordingly, rap1-32E mutants exhibited no sporulation defects (Fig. 3c). The corresponding non-phosphorylatable mutant form of Rap1 (rap1-32A) also did not cause defects in meiotic progression and telomere bouquet behaviour (Fig. 3a,b,c). Western blot analysis from meiotic cell extracts confirmed that the mutant forms of Rap1 were stably expressed, and the phospho-modification of Rap1$32 \mathrm{~A}$ was significantly reduced (Additional file 2). Finally, our yeast two-hybrid assay confirmed that the Bqt1/2 binding domain of Rap1 falls within 216-388 amino acids, and introduced cluster mutations did not affect its interaction with the Bqt1-2 complex (Fig. 3d).

Suspecting that some phospho-modifications might remain unidentified in our study, five additional serine and threonine residues (S-317, T-321, S-322, T-328 and S-364), along with 12 detected phosphosites within and adjacent to the Bqt1/2 binding domain, were all substituted to glutamate (rap1-17E) or alanine (rap1-17A) (Fig. 3a). However, these mutations also did not cause any defects in meiosis (Fig. 3b,c). Thus, we conclude that accumulation of negative charge at the Bqt1-2 binding domain of Rap1 does not affect its ability to form the bouquet.

Because rap1-32A and rap1-32E bear mutations within the binding domain of the telomerase negative regulator Poz1 [21], we checked whether telomere length regulation 


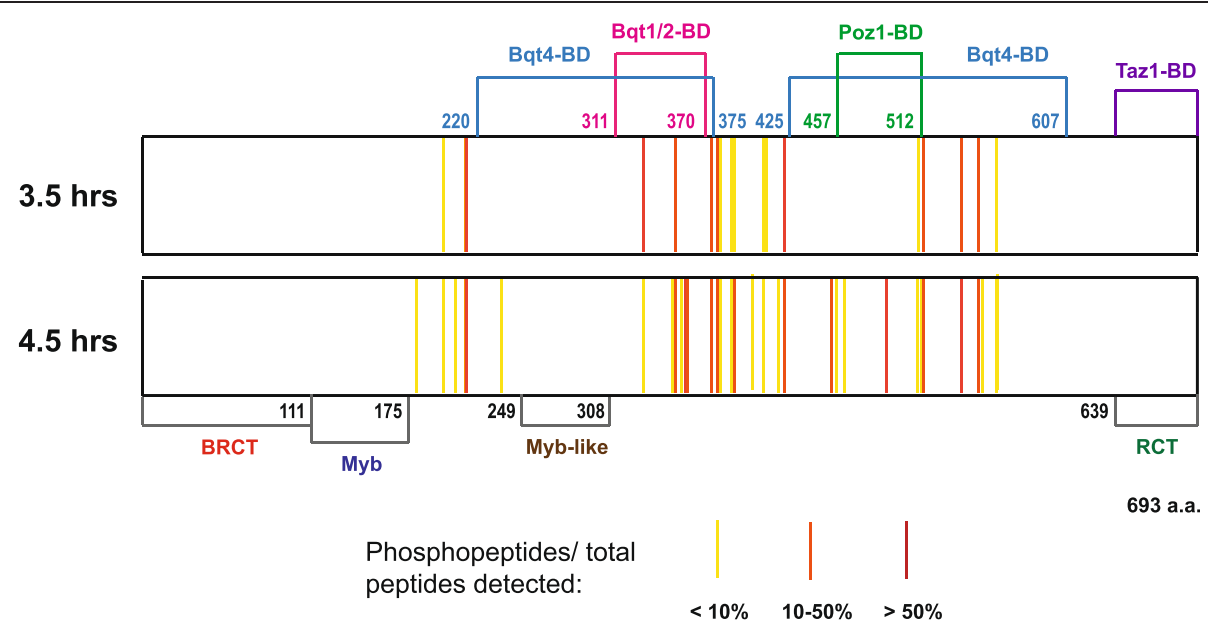

Fig. 2 Domain organization and schematic of phosphorylation sites of Rap1 protein detected at 3.5 hr and 4.5 hr into meiosis. Phosphorylation sites are highlighted as bars with a colour code (yellow, less than 10\%; orange, 10-50\%; and red, over 50\%). Protein interaction domains are indicated above and the structural domains are shown at the bottom. BRCT, BRCA1 C-terminus domain; Myb domain, Myb-like domain; RCT, Rap1 C-terminus domain

was impaired in the rap1 phospho-mutants. Since Cterminus tagging of Rap1 impaired telomere length homeostasis (Fig. 3e), the PK epitope tag was fused to the N-terminus. Although phosphomimetic forms of the Rap1 protein (Rap1-32E and 17E) migrate slower than wild-type Rap1, none of the cluster mutations affected protein stability (Fig. 3f). The strains carrying mutant Rap1 maintained their telomere length comparable to that of wildtype (Fig. 3e). Accordingly, all mutants retained their ability to interact with Poz1 by the yeast two-hybrid assay (Fig. 3g). Additionally, both $32 A$ and $32 E$ mutant forms of Rap1 retained the ability to interact with Bqt4 (Fig. 3h). Indeed, telomere localization to the nuclear periphery in interphase was not impaired in rap1-32A and $32 E \mathrm{mu}-$ tants (Additional file 3). Thus, hyper-phosphorylation of Rap1 observed in meiosis does not appear to have a role in telomere bouquet regulation. Furthermore, our mutagenesis analysis suggests that Rap1 is able to resist high negative charge changes without affecting its function in meiosis or telomere length homeostasis.

\section{Intrinsic negative charge of Bqt1/2 binding domain of Rap1 is crucial for telomere bouquet formation}

Rap1 protein is negatively charged, and the Bqt $1 / 2$ binding region is particularly rich in hydrophobic and negatively charged amino acid residues. Some of these negatively charged residues (D-335, D-337, D-338 and E-342) are well-conserved among fission yeast species (Fig. 4a). Importantly, mutation analysis indicated that Rap1-DD337AA (D337A and D338A mutations) no longer interacts with the Bqt1-2 complex, but retains its ability to interact with Bqt4 and Poz1 in yeast twohybrid assay (Fig. 3d,g,h).

To study the function of Rap1-DD337AA, endogenous rap1 was mutated and fused to YFP. Accordingly, rap1$D D 337 A A$ mutants were defective in sporulation (Figs. 3c and $4 \mathrm{~b})$. Live cell imaging of the mutant showed that Rap1-DD337AA localized to telomeres, as determined by co-localization to Taz1 (Fig. 4c), but did not cluster at the SPB in meiotic prophase (Fig. 4d). Furthermore, in many cases the SPB was destabilized and detached from the nucleus and, as a consequence, aberrant chromosome segregation was observed (Fig. 4d). These meiotic phenotypes are characteristic of rap1 $\Delta$ mutants as well as the bouquet-defective mutants [5]. However, Rap1DD337AA was stably expressed and telomere length of the $\operatorname{rap1-DD337AA}$ mutant was the same as that of wild-type (Fig. 3e,f). Additionally, telomeres of the mutant cells were retained at the nuclear periphery in interphase (Additional file 3). Thus, $\operatorname{rap1-DD337AA}$ is a meiosisspecific loss-of-function mutation, and negatively charged aspartates at positions 337 and 338 are crucial for bouquet formation.

\section{Discussion}

In this study we have shown that the level of phosphorylation of Rap1 gradually increases during the course of meiotic prophase, peaking in meiosis I. The SPB is known to recruit a number of kinases and phosphatases that modify its subunits, and these modifications play a critical role in regulating mitotic commitment and meiotic progression [22-25]. Since telomere heterochromatin comes into close contact with the meiotic SPB during 


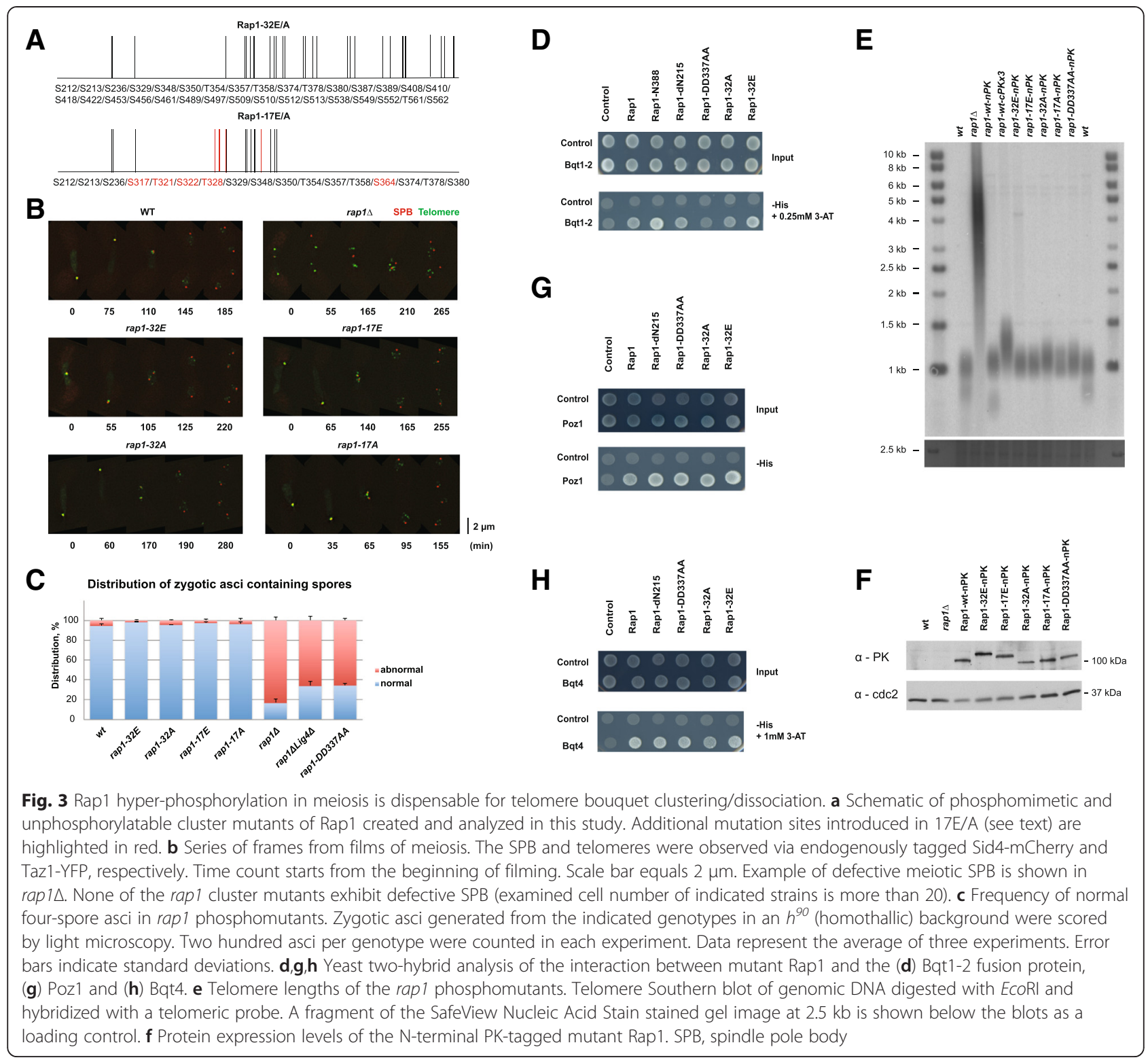

chromosomal bouquet configuration, the telomere-bound proteins are likely to be exposed to these kinases. Additionally, DNA damage checkpoint kinases are activated during meiotic recombination [26]. In fact, some phosphorylations originate from telomere-associated kinases as indicated by reduced shifted bands of Rap1 at 4-4.5 hr in the absence of Taz1 (Additional file 4). Nevertheless, our study suggests that the hyper-phosphorylation of Rap1 observed during meiosis is not directly involved in the regulation of the bouquet. Rap1 can withstand significant charge changes that do not affect interactions with its binding partners and its function in meiosis. Hence, rather than a functional regulatory protein, meiotic Rap1 appears to be a 'scaffolding' protein, that is targeted by multiple kinases. Interestingly, while mutating 32 phosphorylation sites did not alter Rap1 function, mutation of only two highly conserved residues (D-337 and D-338) disrupted its ability to bind to the Bqt1-2 complex, causing pronounced defects in telomere clustering and chromosome segregation. In contrast, the binding partners Bqt1 and Bqt2 are positively charged, suggesting that Rap1 binds to the Bqt1-2 complex through hydrogen bonding interactions. Thus, we speculate that negatively charged residues at Rap1's interaction surfaces are evolutionarily conserved in order to retain affinity under shifts in charge occurring throughout meiotic prophase.

Rap1 is also highly phosphorylated in the mitotic cell cycle, particularly in M-phase. Among the phosphorylation sites reported for mitotic Rap1, five phosphorylation sites (S-213, T-378, S-422, S-456 and S-513) were 


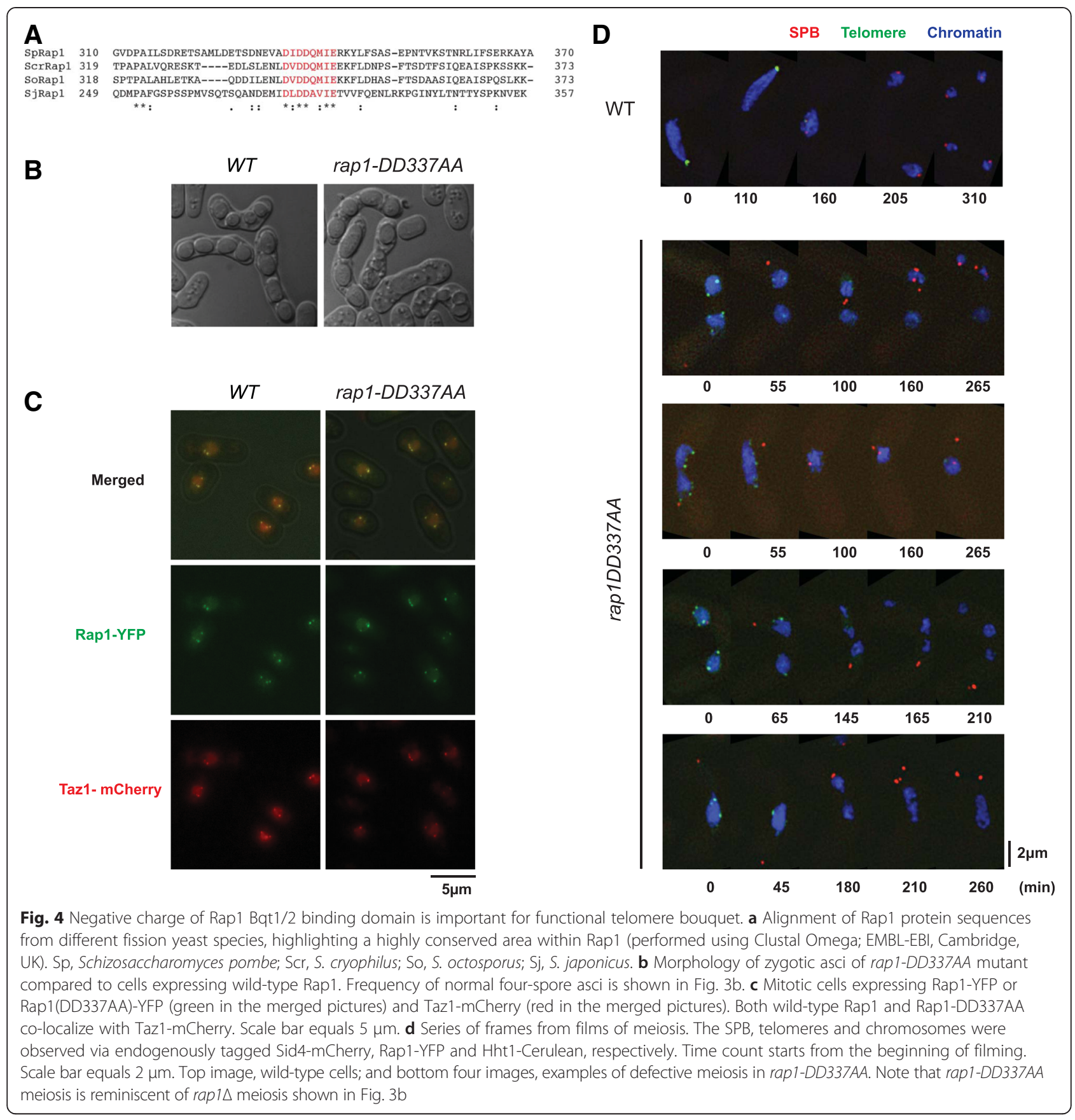

shown to have an inhibitory effect on Rap1-Bqt4 interaction, which was demonstrated by the phosphomimetic rap1-5D/5E mutants [15]. In mitosis, Cdc2 phosphorylates three of these sites in order to temporarily release telomeres from the nuclear envelope. This mechanism assists faithful chromosome segregation in anaphase. However, rap1-5D and rap1-5E mutants do not have any sporulation defects [15], which suggests that the bouquet is intact. This is surprising since the Rap1-Bqt4 interaction is required for telomere clustering in meiosis [8], and raises the possibility that telomeres remain associated with Bqt4 and the SPB via different mechanisms. Notably, four residues of Rap1 including Cdc2 targets were also found to be phosphorylated throughout meiotic prophase in our study (except for S-456, detected only at $4.5 \mathrm{hr}$ ) (Additional file 1). Unlike the Rap1-5E mutant protein, our phosphomimetic cluster mutant Rap1-32E, which includes 5E mutation (Fig. 3a), was able to interact with Bqt4. Thus, we predict that premeiotic hyper-phosphorylation of Rap1 (or particular 
phosphorylations among them) counteracts Cdc2 kinase action to preserve affinity to Bqt4, and thereby maintain telomere localization to the nuclear membrane and bouquet configuration.

\section{Conclusions}

Rap1 hyper-phosphorylation observed during meiotic prophase does not have a direct role in telomere bouquet regulation. Rap1 uses its negatively charged amino acid residues to bind the Bqt1-2 complex. Therefore the interaction is not affected by changes in net charge caused through progressive hyper-phosphorylation.

\section{Methods}

\section{Yeast genetics and plasmids}

The genotypes of the strains used for this study are listed in Additional file 5. All media and supplements were purchased from Formedium $^{\text {tix }}$ (Hunstanton, UK). Fission yeast was grown at $32^{\circ} \mathrm{C}$ in standard rich media (YES) unless indicated. Epitope tag insertion at the C-terminus was described previously [27]. A plasmid for N-tagged rap1 was constructed by cloning the rap1 gene, including 1,360 bases of the upstream and 1,500 bases of the downstream regions, with a primer set including a KpnI site. The start codon of rap1 $1^{+}$was replaced with a single V5 (PK) sequence. A kanMX6 cassette was inserted 700 bases upstream of the gene. The resulting plasmid pRapla-nPK was digested with $K p n I$ and replaced the $\mathrm{ura}_{4}{ }^{+}$cassette at the rap 1 gene locus in rap $1 \Delta$ cells. The transformants were backcrossed with a wild-type strain and cultured for 2 weeks before analysis of telomere length. To generate rap1 mutants, cluster-mutated rap1 gBlocks were synthesized (Integrated DNA Technologies, Coralville, IA, USA) and replaced wild-type rap $^{+}$in the pRapla-nPK plasmid and the yeast two-hybrid pGAD and pGBK plasmids.

\section{Yeast two-hybrid assays}

The assay was conducted according to the Matchmaker Gold Yeast Two-Hybrid System manual (Clontech, Mountain View, CA, USA). Expression vectors for the GAL binding domain (BD) fused proteins and the GAL activation domain (AD) fused proteins were generated by subcloning of the indicated cDNAs into pGBK and pGAD, respectively. Expression of $B D$ and $A D$ fused proteins were confirmed by Western blotting using antimyc and anti-HA antibodies, respectively. To express Bqt1 and Bqt2 together in the yeast two- hybrid plasmids, $b q t 2^{+}$cDNA was inserted at the start codon of the $b q t 1^{+}$cDNA sequence; the resulting plasmid was named pGBK-Bqt2-1.

\section{pat1-114 synchronization}

Cells were first cultured in YE media at $26^{\circ} \mathrm{C}$ overnight until late stationery phase, then transferred to EMM media containing a nitrogen source and incubated for $24 \mathrm{hr}$ until mid-log phase (OD $=0.5-0.7$ ). The cells were next transferred to EMM media without a nitrogen source (EMM-N) by filtering, and cells were incubated for another 15-16 hr to arrest cells at G1 phase. To inactivate the pat1 kinase gene and induce meiosis, the temperature was shifted up to $34^{\circ} \mathrm{C}$, cultures were supplemented with one-fifth volume of EMM media prewarmed to $34^{\circ} \mathrm{C}$, and meiotic fractions were collected at the required time point.

\section{Western blotting and Phos-tag gel}

Whole-cell protein extracts prepared using a trichloroacetic acid method were separated by SDS-PAGE using 10\% acrylamide gels. Western blotting was performed with antiV5 peptide (Bio-Rad, Hercules, CA, USA), anti-Cdc2 (Santa Cruz Biotechnology, Dallas, TX, USA) and anti-Cdc13 (Santa Cruz Biotechnology) antibodies following a standard protocol. For detection of phosphorylated Rap1 forms, $7.5 \%$ acrylamide gels were supplemented with $25 \mu \mathrm{M}$ PhosTag ligand (AAL-107; NARD Institute, Amagasaki, Japan) and $50 \mu \mathrm{M} \mathrm{MnCl}_{2}$, according to the protocol [19]. A Phos-tag gel was treated with $1 \mathrm{mM}$ EDTA prior to transfer. Two technical replicates of the Western blotting image in Fig. 1c are shown in Additional file 6.

\section{Telomere Southern blotting}

Southern blotting was performed as described previously [27]. Equal amounts of EcoRI-digested DNA fragments were separated on a $1 \%$ agarose gel and subjected to Southern blotting with a telomere probe.

\section{Preparation of cell extracts and immunoprecipitation for mass spectrometry}

Six litres of synchronous meiotic cells expressing Rap1$3 \mathrm{xPK}$ were harvested at $3.5 \mathrm{hr}$ (early prophase) or $4.5 \mathrm{hr}$ (late prophase) following the pat1-114 synchronization protocol as described above. Rap1-3xPK was immunoprecipitated from native cell extracts in RIPA buffer (50 mM Tris- $\mathrm{HCl}, \mathrm{pH} 8 ; 150 \mathrm{mM} \mathrm{NaCl} ; 1 \% \mathrm{NP}-40$; $0.5 \%$ sodium deoxycholate, and $0.1 \%$ SDS), supplemented with 1X PhosSTOP (Roche, Basel, Switzerland); $1 \times$ cOmplete EDTA-free protease inhibitor (Roche); $1 \mathrm{mM}$ PMSF, $1 \mathrm{mM}$ DTT, $0.1 \mathrm{ng} / \mathrm{mL}$ MG132 (Sigma-Aldrich, St Louis, MO); and $10 \mathrm{U} / \mathrm{mL}$ TURBO DNase (Ambion, Life Technologies, Carlsbad, CA, USA), using Dynabeads M270 epoxy (Life Technologies) pre-coated with anti-V5 peptide antibodies.

\section{Phosphatase treatment of native cell extracts}

Native whole-cell extracts were prepared using modified HB buffer (50 mM HEPES-KOH, pH 7.5; $140 \mathrm{mM}$ $\mathrm{NaCl}, 0.1 \% \mathrm{NP}-40 ; 1 \mathrm{mM} \mathrm{MnCl} 2$ ). Then $20 \mu \mathrm{l}$ of lysate was treated with $4 \mu \mathrm{l}$ of lambda-phosphatase; $10 \mu \mathrm{l}$ of 
phosphatase inhibitors; both phosphatase and inhibitors; or water as a control. The samples were incubated at $30^{\circ} \mathrm{C}$ for 1 hour before being separated on SDSPAGE gel.

Further details about microscopy and mass spectrometry methods can be found in Additional file 7. A list of phosphopeptides detected by mass spectrometry is shown in Additional file 8.

\section{Open access of data}

The mass spectrometry proteomics data have been deposited to the ProteomeXchange consortium [1] via the PRIDE partner repository with the dataset identifier PXD001841.

\section{Additional files}

\section{Additional file 1: List of Rap1 phosphorylation sites identified in} the study.

Additional file 2: Western blot images of TCA-extracted Rap1 proteins from meiotic cells. Indicated cells were induced to undergo meiosis using the pat1-114 mutation, and were collected from the $4.5 \mathrm{hr}$ fraction. Cell extracts were separated in regular gradient (left) and Phos-tag SDS-PAGE (right), and subjected to Western blot using anti-PK(V5) antibody. Unlike wild-type meiotic Rap1 that shows multiple bands, Rap1-32A exhibits a single band in the Phos-tag gel, suggesting the phospho-modifications were diminished in this mutant form.

Additional file 3: Localization of telomeres in mitotic interphase. (A) Projected images of cells in mitotic interphase. Taz1-YFP, Sid4-mCherry and Bqt3-Cerulean were used to visualize telomeres, spindle pole body and the membrane, respectively. White and grey arrows indicate examples of telomeres localized at the nuclear periphery and away from the nuclear envelope, respectively. Scale bar equals $5 \mu \mathrm{m}$. (B) Percentages of telomeres localized at the nuclear periphery in interphase cells. Cells containing one nucleus and one Sid4-mCherry focus (not dissociated) were selected as mitotic interphase cells. Telomeres were scored as localized at the nuclear periphery when the distance between the brightest Taz1-YFP focus and Bqt3-Cerulean-labeled nuclear membrane was less than $0.4 \mu \mathrm{m}$ using SoftWoRks (Applied Precision; GE Healthcare, Chalfont St Giles, UK). (C) Middle z-section image of the cells in mitotic interphase. Example of telomeres detached from the nuclear envelope (left) and telomeres localized at the nuclear periphery (right). Scale bar equals $5 \mu \mathrm{m}$.

Additional file 4: Reduced phosphorylation of Rap1 in $\operatorname{taz} 1 \Delta$. Separation of phosphorylated Rap1-3xPK on a Phos-tag gel from taz1 $\Delta$ synchronized meiotic cell fractions at indicated time. Anti-Cdc2 (CDK) and anti-Cdc13 (Cyclin B) antibodies were used as a loading control and meiosis synchronicity marker, respectively. Note that Taz1 is required for Rap1 localization at telomeres.

Additional file 5: Fission yeast strain list.

Additional file 6: Technical triplicates of Phos-tag gel related to Fig. 1d.

Additional file 7: Mass spectrometry and microscopy methods. Additional file 8: Mass spectrometry data for identified phosphopeptides.

\section{Abbreviations}

AD: Activation domain; BD: Binding domain; SPB: Spindle pole body.

\section{Competing interests}

The authors declare that they have no competing interests.

\section{Authors' contributions}

HA carried out most of the experiments. HA and KT designed the study and wrote manuscript. SS performed yeast two-hybrid analysis. SRP assisted with yeast two-hybrid assays. VM assisted with live cell imaging. CAA supported yeast genetics analysis. HA, KT, SS and VM generated strains and plasmids. All authors read and approved the final manuscript.

\section{Acknowledgments}

We thank the Proteomics and Metabolomics Core Facility at the Institute for Cancer Research (ICR) for mass spectrometry analysis of the samples and technical advice on the sample preparation. We thank Junko Kanoh (Osaka University, Japan) for critical reading before submission. This work is supported mainly by the European Research Council (281722-HRMCB) and partly by Cancer Research UK (C36439/A12097) and the Cancer Research UK - UCL Centre.

Received: 5 March 2015 Accepted: 4 June 2015

Published online: 10 June 2015

\section{References}

1. Jain D, Cooper JP. Telomeric strategies: means to an end. Annu Rev Genet. 2010;44:243-69.

2. Harper L, Golubovskaya I, Cande WZ. A bouquet of chromosomes. J Cell Sci. 2004:117:4025-32.

3. Scherthan H. A bouquet makes ends meet. Nat Rev Mol Cell Biol. 2001;2:621-7.

4. Niwa O, Shimanuki M, Miki F. Telomere-led bouquet formation facilitates homologous chromosome pairing and restricts ectopic interaction in fission yeast meiosis. EMBO J. 2000;19:3831-40.

5. Tomita K, Cooper JP. The telomere bouquet controls the meiotic spindle. Cell. 2007;130:113-26.

6. Chikashige $Y$, Ding DQ, Funabiki H, Haraguchi T, Mashiko S, Yanagida M, et al. Telomere-led premeiotic chromosome movement in fission yeast. Science. 1994;264:270-3.

7. Chikashige Y, Tsutsumi C, Yamane M, Okamasa K, Haraguchi T, Hiraoka Y Meiotic proteins bqt1 and bqt2 tether telomeres to form the bouquet arrangement of chromosomes. Cell. 2006;125:59-69.

8. Chikashige Y, Yamane M, Okamasa K, Tsutsumi C, Kojidani T, Sato M, et al. Membrane proteins Bqt3 and -4 anchor telomeres to the nuclear envelope to ensure chromosomal bouquet formation. J Cell Biol. 2009;187:413-27.

9. Chikashige $Y$, Hiraoka $Y$. Telomere binding of the Rap1 protein is required for meiosis in fission yeast. Curr Biol. 2001;11:1618-23.

10. Cooper JP, Watanabe Y, Nurse P. Fission yeast Taz1 protein is required for meiotic telomere clustering and recombination. Nature. 1998;392:828-31.

11. Kanoh J, Ishikawa F. spRap1 and spRif1, recruited to telomeres by Taz1, are essential for telomere function in fission yeast. Curr Biol. 2001:11:1624-30.

12. Klutstein M, Fennell A, Fernandez-Alvarez A, Cooper JP. The telomere bouquet regulates meiotic centromere assembly. Nat Cell Biol. 2015;17:458-69.

13. Fennell A, Fernandez-Alvarez A, Tomita K, Cooper JP. Telomeres and centromeres have interchangeable roles in promoting meiotic spindle formation. J Cell Biol. 2015;208:415-28.

14. Tomita $K$, Bez C, Fennell A, Cooper JP. A single internal telomere tract ensures meiotic spindle formation. EMBO Rep. 2013;14:252-60.

15. Fujita I, Nishihara Y, Tanaka M, Tsujii H, Chikashige Y, Watanabe Y, et al. Telomere-nuclear envelope dissociation promoted by Rap1 phosphorylation ensures faithful chromosome segregation. Curr Biol. 2012;22:1932-7.

16. Chikashige Y, Kurokawa R, Haraguchi T, Hiraoka Y. Meiosis induced by inactivation of Pat1 kinase proceeds with aberrant nuclear positioning of centromeres in the fission yeast Schizosaccharomyces pombe. Genes Cells. 2004;9:671-84

17. Bahler J, Schuchert P, Grimm C, Kohli J. Synchronized meiosis and recombination in fission yeast: observations with pat1-114 diploid cells. Curr Genet. 1991;19:445-51.

18. Kanoh J. Release of chromosomes from the nuclear envelope: a universal mechanism for eukaryotic mitosis? Nucleus. 2013;4:100-4.

19. Kinoshita E, Kinoshita-Kikuta E, Takiyama K, Koike T. Phosphate-binding tag, a new tool to visualize phosphorylated proteins. Mol Cell Proteomics. 2006;5:749-57.

20. Fujita I, Tanaka M, Kanoh J. Identification of the functional domains of the telomere protein Rap1 in Schizosaccharomyces pombe. PLoS One. 2012; , e49151. 
21. Miyoshi T, Kanoh J, Saito M, Ishikawa F. Fission yeast Pot1-Tpp1 protects telomeres and regulates telomere length. Science. 2008;320:1341-4.

22. Funaya C, Samarasinghe S, Pruggnaller S, Ohta M, Connolly Y, Muller J, et al. Transient structure associated with the spindle pole body directs meiotic microtubule reorganization in S. pombe. Curr Biol. 2012;22:562-74.

23. Kim S, Meyer R, Chuong H, Dawson DS. Dual mechanisms prevent premature chromosome segregation during meiosis. Genes Dev. 2013;27:2139-46.

24. Ohta M, Sato M, Yamamoto M. Spindle pole body components are reorganized during fission yeast meiosis. Mol Biol Cell. 2012;23:1799-811.

25. Grallert A, Boke E, Hagting A, Hodgson B, Connolly Y, Griffiths JR, et al. A PP1-PP2A phosphatase relay controls mitotic progression. Nature. 2015;517:94-8.

26. Shimada M, Nabeshima K, Tougan T, Nojima H. The meiotic recombination checkpoint is regulated by checkpoint rad + genes in fission yeast. EMBO $\mathrm{J}$. 2002;21:2807-18.

27. Armstrong CA, Pearson SR, Amelina H, Moiseeva V, Tomita K. Telomerase activation after recruitment in fission yeast. Curr Biol. 2014;24:2006-11.

\section{Submit your next manuscript to BioMed Central and take full advantage of:}

- Convenient online submission

- Thorough peer review

- No space constraints or color figure charges

- Immediate publication on acceptance

- Inclusion in PubMed, CAS, Scopus and Google Scholar

- Research which is freely available for redistribution 